\title{
Enthesitis-Related Arthritis: Time to Re-define?
}

\author{
Angela R. Bryan • C. Egla Rabinovich
}

Published online: 4 November 2014

(C) Springer Science+Business Media New York 2014

\begin{abstract}
Juvenile idiopathic arthritis (JIA) is the most common rheumatic disease in childhood. Enthesitis-related arthritis (ERA) is one of the seven JIA subtypes classified by the International League of Associations for Rheumatology (ILAR). Due to inclusion and exclusion criteria, a pitfall of the ERA category is that it does not include all subsets of juvenile spondyloarthropathy, with many children ending up in the undifferentiated category. The ERA nomenclature also does not have a method for distinguishing between axial and peripheral disease, two phenotypes which vary in presentation and treatment requirements. This distinction is very important given the overall poor prognosis seen in ERA patients, specifically in those with axial involvement. Since axial involvement is more common and presents earlier than previously thought in ERA, the pediatric rheumatology community should develop more accurate and sensitive classification criteria based on disease course to assist in improving timely diagnosis and appropriate management.
\end{abstract}

Keywords Juvenile idiopathic arthritis · Enthesitis-related arthritis $\cdot$ Spondyloarthropathy $\cdot$ Juvenile ankylosing spondylitis · Juvenile rheumatoid arthritis $\cdot$ Juvenile psoriatic arthritis

\section{Introduction}

Nomenclature of the chronic arthritides of childhood has undergone evolution over time but remains confusing to the

This article is part of the Topical Collection on Pediatric Rheumatology

A. R. Bryan · C. E. Rabinovich $(\bowtie)$

Division of Pediatric Rheumatology, Duke University Medical

Center, Duke University School of Medicine, Durham, NC 27710,

USA

e-mail: egla.rabinovich@duke.edu nonexperienced and controversial among experts. Enthesitisrelated arthritis (ERA) is one of the seven juvenile idiopathic arthritis (JIA) subtypes classified by the International League of Associations for Rheumatology (ILAR). The ILAR criteria are meant to encompass most children with disease previously classified under juvenile spondyloarthropathy (JSpA), syndrome of seronegative enthesitis and arthritis (SEA), or juvenile ankylosing spondylitis (JAS). One drawback of the ERA category is that it does not include all of the subsets of juvenile spondyloarthropathy and specifically excludes juvenile psoriatic arthritis (JPsA). It also does not distinguish between the two phenotypes of ERA, axial and peripheral disease. This distinction is vital given differing response to medications and the overall prognosis. Axial disease, at times asymptomatic, is more common earlier in the disease process than previously thought. This phenotype of ERA with axial involvement may require a different approach to management compared to peripheral disease to achieve a therapeutic response and halt progressive damage. The pediatric rheumatology community should address these shortcomings of the ILAR classification criteria in order to develop more accurate and sensitive classification criteria and thereby assist in improving research, timely diagnosis, and appropriate management.

\section{Classification Criteria}

Juvenile Idiopathic Arthritis

JIA, the current classification system used by most, consists of a heterogeneous group of diseases whose mutual characteristic is chronic inflammatory arthritis. Older American College of Rheumatology (ACR) criteria defined three subsets of juvenile rheumatoid arthritis (JRA): pauciarticular, polyarticular, and 
systemic. Because of the understanding that the inflammation of ankylosing spondylitis may start in childhood, in 1982, Rosenberg and Petty proposed criteria for syndrome of SEA [1]. SEA delineated a group characterized by the presence of enthesitis with arthralgia or arthritis who had negative rheumatoid factor and antinuclear antibody. The authors hoped in characterizing SEA they could identify those children who would develop spondyloarthritis over time. In 1997, ILAR proposed classification criteria for JIA replacing JRA in the USA and juvenile chronic arthritis (JCA) in Europe. Modifications in 2004 resulted in the current ILAR criteria, which classify JIA into seven subtypes in an effort to create more consistency within the groups [2] (see Table 1).

\section{Enthesitis-Related Arthritis}

ERA is one of the ILAR subtypes, encompassing $11-16 \%$ of all children with JIA [3]. Enthesitis is defined as inflammation of the attachment sites of tendon, ligament, capsule, or fascia into bone, with the classic clinical presentation of Achilles tendonitis and enthesitis (Fig. 1). ERA classification requires the presence of arthritis and enthesitis or either arthritis or enthesitis alone if at least two of the following are present: sacroiliac joint tenderness, inflammatory spinal pain, HLAB27 positivity, male sex with onset of symptoms after 6 years of age, or a family history of HLA-B27 disease in a first- or second-degree relative. Exclusion from the ERA subtype occurs in the presence of psoriasis, often placing those children with psoriasis and features of ERA in the undifferentiated

Table 1 International League of Associations for Rheumatology Juvenile Idiopathic Arthritis Classification Criteria

\begin{tabular}{|c|c|c|}
\hline Category & Definition & Exclusions (see below) \\
\hline Systemic & $\begin{array}{l}\text { Arthritis in } \geq 1 \text { joints with, or preceded by, fever of at least } 2 \text { weeks in duration that is } \\
\text { documented to be daily ("quotidian") for at least } 3 \text { days and accompanied by } \geq 1 \\
\text { of the following: } \\
\text { 1. Evanescent (nonfixed) erythematous rash } \\
\text { 2. Generalized lymph node enlargement } \\
\text { 3. Hepatomegaly and/or splenomegaly } \\
\text { 4. Serositis }\end{array}$ & $\mathrm{a}, \mathrm{b}, \mathrm{c}, \mathrm{d}$ \\
\hline Oligoarthritis & $\begin{array}{l}\text { Arthritis affecting } 1-4 \text { joints during the first } 6 \text { months of disease } \\
\text { Two subcategories: } \\
\text { 1. Persistent oligoarthritis - affecting } \leq 4 \text { joints throughout the disease course } \\
\text { 2. Extended oligoarthritis - affecting }>4 \text { joints after the first } 6 \text { months of disease }\end{array}$ & $\mathrm{a}, \mathrm{b}, \mathrm{c}, \mathrm{d}, \mathrm{e}$ \\
\hline Polyarthritis (RF-negative) & Arthritis affecting $\geq 5$ joints during the first 6 months of disease; a test for $R F$ is negative & $\mathrm{a}, \mathrm{b}, \mathrm{c}, \mathrm{d}, \mathrm{e}$ \\
\hline Polyarthritis (RF-positive) & $\begin{array}{l}\text { Arthritis affecting } \geq 5 \text { joints during the first } 6 \text { months of disease; } \geq 2 \text { tests for } R F \\
\text { at least } 3 \text { months apart during the first } 6 \text { months of disease are positive }\end{array}$ & $\mathrm{a}, \mathrm{b}, \mathrm{c}, \mathrm{e}$ \\
\hline Psoriatic arthritis & $\begin{array}{l}\text { Arthritis and psoriasis, or arthritis and at least } 2 \text { of the following: } \\
\text { Dactylitis } \\
\text { Nail pitting or onycholysis } \\
\text { Psoriasis in a first-degree relative }\end{array}$ & $\mathrm{b}, \mathrm{c}, \mathrm{d}, \mathrm{e}$ \\
\hline Enthesitis-related arthritis & $\begin{array}{l}\text { Arthritis and enthesitis, or arthritis or enthesitis with at least } 2 \text { of the following: } \\
\text { 1. Presence of or a history of sacroiliac joint tenderness and/or inflammatory lumbosacral pain } \\
\text { 2. Presence of HLA-B } 27 \text { antigen } \\
\text { 3. Onset of arthritis in a male }>6 \text { years old } \\
\text { 4. Acute (symptomatic) anterior uveitis } \\
\text { 5. History of ankylosing spondylitis, enthesitis-related arthritis, sacroiliitis with } \\
\text { inflammatory bowel disease, Reiter's syndrome, or acute anterior } \\
\text { uveitis in a first-degree relative }\end{array}$ & $\mathrm{a}, \mathrm{d}, \mathrm{e}$ \\
\hline Undifferentiated arthritis & Arthritis that fulfills criteria in no category or in $\geq 2$ of the above categories & \\
\hline
\end{tabular}

Refer also to reference [2]. Exclusions are as follows:

a. Psoriasis or a history of psoriasis in the patient or a first-degree relative

b. Arthritis in an HLA-B27-positive male beginning after the sixth birthday

c. Ankylosing spondylitis, enthesitis-related arthritis, sacroiliitis with inflammatory bowel disease, Reiter's syndrome, or acute anterior uveitis, or a history of one of these disorders in a first-degree relative

d. Presence of immunoglobulin M rheumatoid factor (RF) on at least two occasions at least 3 months apart

e. Presence of systemic JIA in the patient 


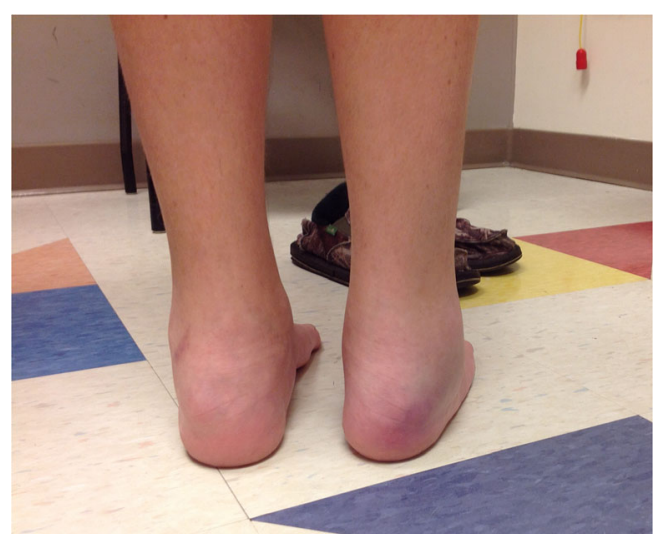

Fig. 1 A 15-year-old boy with HLA-B27 positivity, bilateral ankle arthritis, diffuse Achilles tendonitis, and enthesitis

category. Similarly, if a child with psoriasis and arthritis is male with HLA-B27 positivity, or has a family history of spondyloarthritis, they are excluded from the psoriatic category and placed in the undifferentiated arthritis category.

Juvenile Spondyloarthropathy

While the JIA classification criterion was evolving, there was a different nomenclature often used to refer to those at risk for axial spine inflammation. The term juvenile spondyloarthropathy (JSpA) refers to a group of inflammatory conditions in children characterized by arthritis, certain genetic associations (including presence of HLA-B27), enthesitis, and increased risk of inflammatory back pain $[4,5]$. JSpA includes SEA, ERA, JAS (used when there is radiographic evidence of axial inflammation), reactive arthritis (ReA), JPsA, and undifferentiated arthritis. These patients are characterized by asymmetric, predominantly oligoarticular lower extremity arthritis along with enthesopathy, a family history of similar disease, negative rheumatoid factor (RF), and positive HLA-B27 antigen. Predominance in males and an older age of onset further characterized this group. In adults, the prototypical disease is ankylosing spondylitis. It was thought that axial disease was uncommon in JSpA at presentation but could develop much later in the disease process. JIA was meant to incorporate JSpA in the ILAR nomenclature, but JSpA is still commonly used and can lead to confusion $[1,4,5,6 \bullet, 7]$.

\section{Juvenile Psoriatic Arthritis}

Since the inception of the ILAR criteria, there has been debate regarding several aspects of the ERA subtype. JPsA was specifically excluded from the ERA subtype and placed into a separate category. A study evaluating JPsA has shown two distinct phenotypic groups of patients [8]. There is a younger ( $<5$ years of age) group with peak age of onset of 2 years whose arthritis is more likely to exhibit dactylitis and small joint involvement. These children are more likely to be female and have a positive anti-nuclear antibody (ANA). An older male group tends to present with more enthesitis, axial disease, and persistent oligoarthritis [8]. Older male pediatric patients who meet the current criteria for JPsA actually share more similarities with ERA patients without psoriasis $[8,9 \bullet \bullet$. In a study examining disease manifestations and course in a group of children with JPsA, they did not appear to form a distinct subgroup of patients; rather, they fell in to one of existing ILAR classifications, suggesting that this category may not be as useful as originally thought [8].

\section{Comparison of Juvenile Spondyloarthropathy Criteria}

Several other classification systems have attempted to categorize children with chronic inflammatory arthritis who fulfill the criteria for ERA. One study compared the SEA criteria, Garmisch-Partenkirchen criteria, atypical spondyloarthropathy criteria, Amor criteria, and European Spondyloarthropathy Study Group (ESSG) criteria for sensitivity and specificity in classifying JSpA [7]. The criteria that are used primarily in children (the SEA criteria, GarmischPartenkirchen criteria, and atypical spondyloarthropathy criteria) were not able to fully differentiate between JSpA and other forms of JIA. The adult criteria, the ESSG and Amor criteria, were able to diagnose definite JAS, but not undifferentiated forms of JSpA. Therefore, none of these systems were found to be ideal and the authors concluded that new criteria need to be developed [7].

\section{Adult Spondyloarthropathy}

Compared to pediatric-onset disease, adult-onset spondyloarthropathy ( $\mathrm{SpA}$ ) has more axial involvement (95 versus $74 \%$ ), more radiographic changes, and more functional limitations [10]. Juvenile-onset spondyloarthropathy tends to have more episodes of acute uveitis and more commonly reached remission in one study [10]. There were no differences between adult- and pediatric-onset disease in gender, HLA-B27 status, ethnicity, tender and swollen joint counts, the presence of enthesitis, or joint replacement or spinal surgery rates [10]. Another study reported similar findings with increased peripheral arthritis and decreased back pain in the juvenile-onset patients but conversely found that patient global scores were worse in juvenile-onset as compared to adultonset disease $[11 \bullet \bullet$.

While there are differences between pediatric- and adultonset spondyloarthropathy, the recent adult criteria updates may inform the development of improved pediatric criteria. The modified New York criteria for adult spondyloarthropathy require 3 months of inflammatory back pain with radiographic evidence of inflammatory spinal changes [12]. With the recognition that radiographic changes are not consistently present in early disease and are possibly more representative of 
damage than active inflammation, new guidelines were published by the Assessment of SpondyloArthritis international Society (ASAS) in 2009 [13]. Adult axial spondyloarthropathy is now defined by those with more than 3 months of back pain either with sacroiliitis on radiographs or MRI and one typical spondyloarthropathy feature, or alternatively by those with a positive HLA-B27 and two typical spondyloarthropathy features. Typical spondyloarthropathy features include inflammatory back pain, arthritis, enthesitis, uveitis, dactylitis, psoriasis, inflammatory bowel disease, positive clinical response to NSAIDs, family history of spondyloarthropathy, positive HLA-B27, and elevated CRP [13]. These updated criteria are assisting medical providers in earlier diagnosis and implementation of therapy.

\section{Axial Versus Peripheral Disease in ERA}

The use of various classification schemes over time to diagnose this subtype of arthritis has made it difficult to discern the natural history of ERA and to target appropriate treatment. Based on recent data, it appears that common theories on the presentation and progression of this disease are not accurate. In the past, axial involvement at diagnosis was felt to be rare while peripheral arthritis and enthesitis were much more commonplace in children $[5,10]$. Children with ERA were expected to develop axial involvement only after prolonged inflammation. However, one study showed that $66-75 \%$ of HLA-B27-positive JCA/JRA patients and 70-90\% SEA patients fulfilled the criteria for ankylosing spondylitis 510 years after the onset of symptoms [14].

Newer evidence suggests that axial involvement occurs much earlier. Two distinct phenotypes for ERA, those with predominantly axial or peripheral disease, appear to be emerging, each with a unique presentation, treatment requirement, and prognosis. Patients with axial ERA tend to be older with an average age of onset of 11 years and 4 months, have HLAB27 positivity ( $83 \%$ ), have hip arthritis along with sacroiliac and lumbar spine symptoms, and more commonly have acute anterior uveitis or inflammatory bowel disease. The peripheral ERA cohort is younger with the average age of 9 years and 8 months, less commonly HLA-B27 positive (49\%), and has more ankle arthritis and enthesitis. The patients with axial disease seem to respond readily to anti-tumor necrosis factor (TNF) medications, a phenomenon also seen repeatedly in the adult literature [15•]. Several studies have shown a lack of efficacy for DMARDs in axial disease [16-18]. Additionally, there is evidence in the adult literature that while symptoms can be well controlled by anti-TNF agents, radiographic progression may be difficult to halt once initiated $[14,19,20]$. This suggests that there may be a window of opportunity in children to intervene early in ERA to avoid chronic axial complications. However, more research is needed to identify those children in whom early treatment would be beneficial.

Increased utilization of MRI has allowed us to detect silent and early sacroiliac disease, particularly when the MRI is enhanced by contrast [21]. One study suggested that axial involvement can be seen in over half of patients with ERA after 2-3 years of disease and can be seen on MRI in $10 \%$ prior to clinical symptoms [15•]. In most ERA patients, the sacroiliac joints are the first area of axial involvement [6•]. Registry data from the Childhood Arthritis and Rheumatology Research Alliance (CARRA) demonstrated that $28 \%$ of children had sacroiliac inflammation at diagnosis [22•]. Other studies have shown similar numbers with inflammatory back pain in 21 out of 59 patients ( $35 \%$ ) within 1 year and 3 months of diagnosis [23•]. It is instructive that all 21 patients had normal plain films, but 17 had sacroiliitis on MRI [23•].

The prevalence of early or silent disease was demonstrated in a study of 143 children diagnosed with spondyloarthropathy [24•]. Fifty three (37\%) of the children had sacroiliitis on physical exam or imaging. Eleven of these 53 patients $(21 \%)$ had silent disease with no back pain, stiffness, or abnormal exam reported. Inflammation was seen on MRI in 7, in plain radiographs in 8 , and on both methods of imaging in 4 [24•]. This is similar to an earlier report that $20 \%$ of JSpA patients have sacroiliitis on MRI despite lack of axial symptoms [25]. Another recent retrospective study revealed that the majority ( $93 \%$ ) of the participating ERA patients had MRI evidence of inflammation in either the sacroiliac joints (78 \%), the lumbar spine (67\%), or both (52\%). The average interval between diagnosis and the MRI was 3.8 years [26••].

\section{Prognosis in ERA}

The literature demonstrates an overall poor prognosis for ERA patients. The very nature of ERA with initially vague and sometimes intermittent symptoms as well as the insensitivity of plain films to early inflammatory sacroiliac disease leads to delays in diagnosis and treatment. Prior to the introduction of biologic medications, ERA had an $18 \%$ probability of remission at a median of 16.5 years, which was less than oligoarticular JIA (54\%) and systemic JIA (47 \%) [27]. Another study from the prebiologic period reported a remission rate of $44 \%$ at 15.3 years [3]. Those who were not in remission had persistent sacroiliac disease (35\%) and reduced spinal flexion (75\%) at 15.3 years of disease, and need for anti-rheumatic drugs ( $45 \%$ ) at 23 years of disease. Predictors of failure to achieve remission included a first-degree relative with ankylosing spondylitis, HLA-DRB $1 * 08$, and ankle arthritis in the first 6 months of disease. Predictors of persistent sacroiliitis included a persistently elevated ESR and hip arthritis in the first 6 months of diagnosis [3]. Additional predictors of continued disease activity at 8 years were HLA-B27 positivity, enthesitis, sacroiliac symptoms, and hip arthritis 
[28•]. A study reviewing inactive disease at 1 year follow-up after starting TNF inhibitors reported similar outcomes for RF-negative polyarthritis (57\%), persistent oligoarticular arthritis $(64 \%)$, and psoriatic arthritis $(60 \%)$ but much lower rates of inactive disease for enthesitis-related arthritis at only $24 \%[29 \cdot]$.

After 15.3 and 23 years of disease, children with ERA had lower levels of physical functioning, poorer physical health, and more pain compared to those with oligoarticular and polyarticular JIA [3]. A prospective study of JRA and JSpA (excluding patients with enthesitis only) also found that patients classified at the time as JAS, SEA, and RF-positive polyarticular JRA had the poorest physical functioning, highest pain, and worst well-being [19]. Studies in the biologic era revealed similar prognoses for these patients [30••, $31 \bullet \bullet$. The CARRA registry encompassing 268 children with ERA had similar results with reports of more frequent pain, with higher pain intensity, and impaired function in ERA compared to other JIA categories. Higher active joint counts and NSAID, biologic, and steroid use predicted worse scores on patient-reported measures on multivariable regression. Active enthesitis, SI tenderness, and NSAID use were associated with elevated pain intensity in ERA [31••].

There is now a case report in the literature of serial radiographic studies of peripheral joints showing radiologic improvement in a child with ERA receiving anti-tumor necrosis factor therapy, supporting the theory that early intervention may affect outcome [32]. More research will need to occur to examine changes of radiographic progression in the axial skeleton.

\section{Conclusion}

The poor prognosis associated with enthesitis-related arthritis is multifactorial. Delayed diagnosis in general leads to delayed treatment initiation. Once the disease is diagnosed, the lack of recognition of early axial inflammation and the inefficacy of certain medication classes for axial disease lead to further delay in appropriate treatment. Sacroiliitis is known to be associated with increased morbidity and mortality in adults. Once axial damage has occurred, our current therapies may not always be able to halt further progression of the disease. The poor prognosis and evidence supporting silent, early axial disease should push the pediatric rheumatology community to develop more accurate and sensitive classification criteria incorporating what are known about different phenotypic manifestations of classic JSpA. Having a diagnosis that would follow a child into adulthood would be ideal. This would be a first step in improving time to diagnosis and developing appropriately targeted therapy early. This is of paramount importance in these children to attempt to achieve disease remission and to avoid future complications and disability.

\section{Compliance with Ethics Guidelines}

Conflict of Interest Angela R. Bryan declares no conflict of interest.

C. Egla Rabinovich declares the receipt of research grants from UCB, Hoffman LaRoche, and AbbieVie, outside the submitted work.

Human and Animal Rights and Informed Consent This article does not contain any studies with human or animal subjects performed by any of the authors.

\section{References}

Papers of particular interest, published recently, have been highlighted as:

- Of importance

•. Of major importance

1. Rosenberg AM, Petty RE. A syndrome of seronegative enthesopathy and arthropathy in children. Arthritis Rheum. 1982;25(9):1041-7.

2. Petty RE et al. International League of Associations for Rheumatology classification of juvenile idiopathic arthritis: second revision, Edmonton, 2001. J Rheumatol. 2004;31(2):390-2.

3. Flato B et al. Long-term outcome and prognostic factors in enthesitis-related arthritis: a case-control study. Arthritis Rheum. 2006;54(11):3573-82.

4. Hofer M. Spondylarthropathies in children - are they different from those in adults? Best Pract Res Clin Rheumatol. 2006;20(2):31528.

5. Colbert RA. Classification of juvenile spondyloarthritis: enthesitisrelated arthritis and beyond. Nat Rev Rheumatol. 2010;6(8):47785 .

6. Ramanathan A, Srinivasalu H, Colbert RA. Update on juvenile spondyloarthritis. Rheum Dis Clin N Am. 2013;39(4):767-88. This is a review of juvenile spondyloarthropathies, including clinical features, genetics and therapeutic options.

7. Kasapcopur $\mathrm{O}$ et al. Evaluation of classification criteria for juvenile-onset spondyloarthropathies. Rheumatol Int. 2005;25(6): 414-8.

8. Stoll ML et al. Patients with juvenile psoriatic arthritis comprise two distinct populations. Arthritis Rheum. 2006;54(11):3564-72.

9.• Butbul Aviel Y et al. Juvenile psoriatic arthritis (JPsA): juvenile arthritis with psoriasis? Pediatr Rheumatol Online J. 2013;11(1):11. A cohort of children with JPSA were examined for unifying clinical features and long term outcomes.

10. O'Shea FD et al. Comparison of clinical and radiographic severity of juvenile-onset versus adult-onset ankylosing spondylitis. Ann Rheum Dis. 2009;68(9):1407-12.

11.• Chen HA et al. Clinical, functional, and radiographic differences among juvenile-onset, adult-onset, and late-onset ankylosing spondylitis. J Rheumatol. 2012;39(5):1013-8. The differences between pediatric and adult onset ankylosing spondylitis examined in a large cohort of Taiwanese patients.

12. van der Linden S, Valkenburg HA, Cats A. Evaluation of diagnostic criteria for ankylosing spondylitis. A proposal for modification of the New York criteria. Arthritis Rheum. 1984;27(4):361-8.

13. Rudwaleit $\mathrm{M}$ et al. The development of Assessment of SpondyloArthritis international Society classification criteria for 
axial spondyloarthritis (part II): validation and final selection. Ann Rheum Dis. 2009;68(6):777-83.

14. Burgos-Vargas R. Juvenile onset spondyloarthropathies: therapeutic aspects. Ann Rheum Dis. 2002;61 Suppl 3:iii33-9.

15. Fisher $\mathrm{C}$ et al. Enthesitis-related arthritis; a new era of understanding. Ann Paediatr Rheum. 2012;1(1):8-16. A review of differences between axial and peripheral ERA.

16. Chen J, Liu C. Is sulfasalazine effective in ankylosing spondylitis? A systematic review of randomized controlled trials. J Rheumatol. 2006;33(4):722-31.

17. Haibel $\mathrm{H}$ et al. No efficacy of subcutaneous methotrexate in active ankylosing spondylitis: a 16-week open-label trial. Ann Rheum Dis. 2007;66(3):419-21.

18. Haibel $\mathrm{H}$ et al. Six months open label trial of leflunomide in active ankylosing spondylitis. Ann Rheum Dis. 2005;64(1):124-6.

19. Selvaag AM et al. Early disease course and predictors of disability in juvenile rheumatoid arthritis and juvenile spondyloarthropathy: a 3 year prospective study. J Rheumatol. 2005;32(6):1122-30.

20. van der Heijde D et al. Assessment of radiographic progression in the spines of patients with ankylosing spondylitis treated with adalimumab for up to 2 years. Arthritis Res Ther. 2009;11(4):R127.

21. Althoff $\mathrm{CE}$ et al. Magnetic resonance imaging of active sacroiliitis: do we really need gadolinium? Eur J Radiol. 2009;71(2):232-6.

22. Weiss PF et al. Enthesitis in an inception cohort of enthesitis-related arthritis. Arthritis Care Res (Hoboken). 2011;63(9):1307-12. Examination of characteristics of an inception cohort of patients with ERA.

23. Pagnini I et al. Early predictors of juvenile sacroiliitis in enthesitisrelated arthritis. J Rheumatol. 2010;37(11). Prospective study of ERA, examining incidence of sacroiliitis and early predictors for development of sacroiliitis.

24. Stoll ML et al. Spondyloarthritis in a pediatric population: risk factors for sacroiliitis. J Rheumatol. 2010;37(11):2402-8. Authors retrospectively examined risk factors for sacroiliitis, finding hip arthritis an important predictor.
25. Bollow $\mathrm{M}$ et al. Use of dynamic magnetic resonance imaging to detect sacroiliitis in HLA-B27 positive and negative children with juvenile arthritides. J Rheumatol. 1998;25(3):556-64.

$26 . \bullet$ Vendhan $\mathrm{K}$ et al. Inflammatory changes of the lumbar spine in children and adolescents with enthesitis-related arthritis: magnetic resonance imaging findings. Arthritis Care Res (Hoboken). 2014;66(1):40-6. MRI abnormalities in the spine are examined and compared to a control population.

27. Minden $\mathrm{K}$ et al. Long-term outcome in patients with juvenile idiopathic arthritis. Arthritis Rheum. 2002;46(9):2392-401.

28. Berntson L et al. HLA-B27 predicts a more chronic disease course in an 8-year followup cohort of patients with juvenile idiopathic arthritis. J Rheumatol. 2013;40(5):725-31. Large incident cohort of children with JIA prospectively examined and clinical characteristics examined and compared in HLA-B27 positive and negative patients.

29. Donnithorne KJ, Cron RQ, Beukelman T. Attainment of inactive disease status following initiation of TNF-alpha inhibitor therapy for juvenile idiopathic arthritis: enthesitis-related arthritis predicts persistent active disease. J Rheumatol. 2011;38(12):2675-81. Retrospective, single site study of JIA patients with examination of attainment of inactive disease, and risk factors for persistent active disease after initiation of anti-TNF therapy.

30.• Boiu $\mathrm{S}$ et al. Functional status in severe juvenile idiopathic arthritis in the biologic treatment era: an assessment in a French paediatric rheumatology referral centre. Rheumatology (Oxford). 2012;51(7): 1285. Disability, pain and functional status examined in a cohort of patients with JIA.

31.• Weiss PF et al. Enthesitis-related arthritis is associated with higher pain intensity and poorer health status in comparison with other categories of juvenile idiopathic arthritis: the Childhood Arthritis and Rheumatology Research Alliance Registry. J Rheumatol. 2012;39(12):2341-51. Pain and health status examined in a large cross sectional study of JIA patients.

32. Tse SM et al. Radiologic improvement of juvenile idiopathic arthritisenthesitis-related arthritis following anti-tumor necrosis factor-alpha blockade with etanercept. J Rheumatol. 2006;33(6):1186-8. 